\title{
Article \\ Graphs Having Most of Their Eigenvalues Shared by a Vertex Deleted Subgraph
}

\author{
Alexander Farrugia \\ Department of Mathematics, G.F. Abela University of Malta Junior College, MSD 1252 Msida, Malta; \\ alex.farrugia@um.edu.mt

\begin{abstract}
Let $G$ be a simple graph and $\{1,2, \ldots, n\}$ be its vertex set. The polynomial reconstruction problem asks the question: given a deck $\mathscr{P}(G)$ containing the $n$ characteristic polynomials of the vertex deleted subgraphs $G-1, G-2, \ldots, G-n$ of $G$, can $\phi(G, x)$, the characteristic polynomial of $G$, be reconstructed uniquely? To date, this long-standing problem has only been solved in the affirmative for some specific classes of graphs. We prove that if there exists a vertex $v$ such that more than half of the eigenvalues of $G$ are shared with those of $G-v$, then this fact is recognizable from $\mathscr{P}(G)$, which allows the reconstruction of $\phi(G, x)$. To accomplish this, we make use of determinants of certain walk matrices of $G$. Our main result is used, in particular, to prove that the reconstruction of the characteristic polynomial from $\mathscr{P}(G)$ is possible for a large subclass of disconnected graphs, strengthening a result by Sciriha and Formosa.
\end{abstract}

check for

updates

Citation: Farrugia, A. Graphs

Having Most of Their Eigenvalues Shared by a Vertex Deleted Subgraph. Symmetry 2021, 13, 1663. https:// doi.org/10.3390/sym13091663

Academic Editor: Jan Cz.

Dobrowolski

Received: 15 July 2021

Accepted: 6 September 2021

Published: 9 September 2021

Publisher's Note: MDPI stays neutral with regard to jurisdictional claims in published maps and institutional affiliations.

Copyright: (C) 2021 by the author. Licensee MDPI, Basel, Switzerland. This article is an open access article distributed under the terms and conditions of the Creative Commons Attribution (CC BY) license (https:// creativecommons.org/licenses/by/ $4.0 /)$.
Keywords: polynomial reconstruction problem; graph walk; eigenvalue; characteristic polynomial; vertex deleted subgraph; disconnected graph

MSC: 05C50; 05C83

\section{Introduction}

Let $G$ be a simple undirected graph, having no loops, no multiple edges and no weighted edges. The vertex set of $G$ is $\mathcal{V}(G)=\{1,2, \ldots, n\}$. The monic characteristic polynomial of $G$ in the variable $x$, denoted by $\phi(G, x)$, is the determinant of $x \mathbf{I}-\mathbf{A}$, where $\mathbf{I}$ is the $n \times n$ identity matrix and $\mathbf{A}$ is the adjacency matrix of $G$. The roots of $\phi(G, x)$ are the eigenvalues of $G$. Since $\mathbf{A}$ is a real and symmetric matrix, the eigenvalues of $G$ are real numbers. An eigenvector $\mathbf{x}$ of an eigenvalue $\lambda$ of $G$ is a nonzero vector satisfying $\mathbf{A x}=\lambda \mathbf{x}$. The eigenspace associated with an eigenvalue $\lambda$ of $G$, denoted by $E(\lambda)$, is the vector space containing the zero vector 0 together with every possible eigenvector of $\lambda$. For any vertex $v$, the graph $G-v$ is the graph obtained from $G$ by removing $v$ and the edges incident to $v$.

In 1973, the polynomial reconstruction problem was posed by Cvetković at the XVIII International Scientific Colloquium held in Ilmenau [1,2]. It asked the following question: Is it true that for $n>2$, the characteristic polynomial $\phi(G, x)$ of a simple graph $G$ on $n$ vertices is determined uniquely by the polynomial deck

$$
\mathscr{P}(G)=\{\phi(G-1, x), \ldots, \phi(G-n, x)\}
$$

of characteristic polynomials of vertex deleted subgraphs of $G$ ? The problem, which was also posed by Schwenk [3] independently of Cvetković, is still open, although it was answered in the affirmative for several classes of graphs. Examples of such graph classes include the class containing graphs whose eigenvalues are bounded below by -2 ; for these graphs, the answer is 'yes' for both the connected [4] and the disconnected cases [5]. Other graph classes for which the problem has an affirmative answer are trees [6], unicyclic graphs [7] and regular graphs [8]. Further results on the reconstruction of $\phi(G, x)$ from 
$\mathscr{P}(x)$ were also put forward for graphs having terminal vertices [9], for bipartite graphs [8] and for disconnected graphs [10].

In this paper, we show that $\mathscr{P}(G)$ has enough information for us to deduce whether or not $\phi(G, x)$ shares more than half of its roots (where multiple roots are counted as many times as their respective multiplicities) with one of the polynomials in $\mathscr{P}(G)$. If more than half of the eigenvalues of $G$ are indeed shared by those of one of its vertex deleted subgraphs $G-v$, say, then $\phi(G, x)$ is shown to be reconstructible from $\mathscr{P}(G)$, even though these common eigenvalues in $G$ and $G-v$ are not known prior to the completion of this reconstruction.

To achieve our goals, we make use of graph walks. A walk in $G$ of length $k$ is a sequence

$$
v_{0}, e_{1}, v_{1}, e_{2}, v_{2}, e_{3}, \ldots, v_{k-1}, e_{k}, v_{k}
$$

where $v_{0}, \ldots, v_{k}$ are vertices of $G, e_{1}, \ldots, e_{k}$ are edges of $G$, and each edge $e_{j}$ in this sequence connects vertices $v_{j-1}$ and $v_{j}$ (neither the vertices nor the edges in this sequence are necessarily distinct). The walk is closed if $v_{0}=v_{k}$. We consider $n$ walk matrices of $G$, $\mathbf{W}_{1}, \mathbf{W}_{2}, \ldots, \mathbf{W}_{n}$, each containing walk enumerations of $G$ that end at vertex $1,2, \ldots, n$ in $G$, respectively. Even though as far as this paper is concerned, these matrices are not themselves reconstructible from $\mathscr{P}(G)$, if for some $v \in \mathcal{V}(G)$, the greatest common divisor of $\phi(G, x)$ and $\phi(G-v, x)$ is of degree more than $\frac{n}{2}$, then we shall be able to deduce enough information about $\mathbf{W}_{v}$ for us to accomplish our task of reconstructing $\phi(G, x)$.

The proof of the main result of this paper is in Section 5, specifically in Theorem 13. Before we present this proof, the next three sections contain results for walk matrices, for companion matrices, and for eigenvalues of both the graph $G$ and for any of its vertex deleted subgraphs. Section 6 illustrates our techniques by successfully reconstructing the characteristic polynomial of two example graphs from their respective polynomial decks. The final section contains a remarkable consequence of our main result, Theorem 14, stating that the polynomial reconstruction problem is settled in the affirmative for a large subclass of disconnected graphs. This result further strengthens a result first obtained by Sciriha and Formosa in [10].

\section{Walk Matrices}

In the literature, a walk matrix $\mathbf{W}_{\mathbf{b}}$ is a matrix of the form

$$
\left(\begin{array}{lllll}
\mathbf{b} & \mathbf{A b} & \mathbf{A}^{2} \mathbf{b} & \cdots & \mathbf{A}^{k-1} \mathbf{b}
\end{array}\right)
$$

where $\mathbf{b}$ is a $0-1$ vector. Usually, $\mathbf{b}$ is taken to be $\mathbf{j}$, the vector of all ones [11-14], but there are exceptions [15-18]. For every $i$ and $j$, the entry in the $i$ th row and $j$ th column of $\mathbf{W}_{\mathbf{b}}$ is equal to the number of walks of length $j-1$ that start from vertex $i$ and end at any vertex in $S$, where $S$ is the subset of $\mathcal{V}(G)$ indicated by the entries in $\mathbf{b}$ that are equal to 1 . It is known (see $[15,19])$ that, for any indicator vector $\mathbf{b}$ and any number of columns $k$ of $\mathbf{W}_{\mathbf{b}}$ we choose the matrix to have, there is a number $r$ such that the rank of $\mathbf{W}_{\mathbf{b}}$ is $k$ for all $k \leq r$ and is $r$ for all $k>r$. For this reason, $\mathbf{W}_{\mathbf{b}}$ is either assumed to have $r$ columns $[11,15,19]$, or $n$ columns $[12,16,18]$.

In this paper, we consider walk matrices $\mathbf{W}_{\mathbf{e}_{v}}, v \in \mathcal{V}(G)$, where $\left\{\mathbf{e}_{1}, \ldots, \mathbf{e}_{n}\right\}$ is the standard $n$ dimensional vector basis for $\mathbb{R}^{n}$. To slightly simplify the notation, henceforth we denote the matrix $\mathbf{W}_{\mathbf{e}_{v}}$ by $\mathbf{W}_{v}$. Moreover, for each vertex $v$, the number of columns of $\mathbf{W}_{v}$ is the minimum possible number of columns such that its rank is maximized; in other words, the number of columns of $\mathbf{W}_{v}$ is the number $r$ described in the previous paragraph. Of course, for distinct vertices $u$ and $v$, the ranks of $\mathbf{W}_{u}$ and $\mathbf{W}_{v}$ may differ. Thus, the $n$ matrices $\mathbf{W}_{1}, \ldots, \mathbf{W}_{n}$ may not have the same number of columns, but for each of them, the rank will stay the same if the number of columns is increased by any number, and will decrease by $s$ if the number of columns is decreased by $s$, for any feasible $s$.

As in the References $[15,16]$, for each walk matrix $\mathbf{W}_{v}$, we consider the Gram matrix of its columns, which is the matrix $\mathbf{H}_{v}=\mathbf{W}_{v}^{\top} \mathbf{W}_{v}$. It is well-known that $\mathbf{H}_{v}$ is a positive 
semidefinite matrix having the same rank as $\mathbf{W}_{v}$ [20]. In this case, since, by definition, $\mathbf{W}_{v}$ has full rank, $\mathbf{H}_{v}$ is invertible and, hence, positive definite. Moreover,

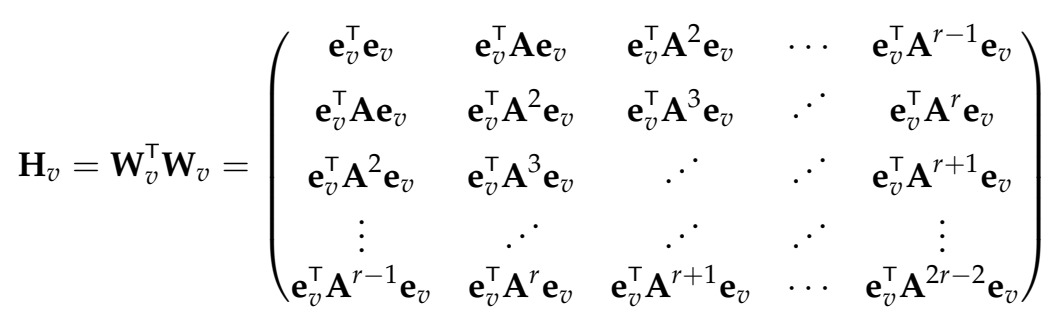

so that, for all $j$ and $k$, the $j k$ th entry of $\mathbf{H}_{v}$ is the number of closed walks of length $j+k-2$ in $G$ that start and end at vertex $v$. Note also that $\mathbf{H}_{v}$ has constant skew diagonals, so it is a Hankel matrix. We denote these walk enumerations by $w_{0}(v), w_{1}(v), \ldots, w_{2 r-2}(v)$ or by $w_{0}, w_{1}, \ldots, w_{2 r-2}$ if the vertex $v$ in question is inferable from the context.

Thus, for any $t$, the matrix

$$
\left(\begin{array}{ccccc}
w_{0} & w_{1} & w_{2} & \cdots & w_{t-1} \\
w_{1} & w_{2} & w_{3} & . \cdot & w_{t} \\
w_{2} & w_{3} & . \cdot & . \cdot & w_{t+1} \\
\vdots & . \cdot & . \cdot & . \cdot & \vdots \\
w_{t-1} & w_{t} & w_{t+1} & \cdots & w_{2 t-2}
\end{array}\right)
$$

has the same rank as the matrix

$$
\left(\begin{array}{lllll}
\mathbf{e}_{v} & \mathbf{A} \mathbf{e}_{v} & \mathbf{A}^{2} \mathbf{e}_{v} & \cdots & \mathbf{A}^{t-1} \mathbf{e}_{v}
\end{array}\right)
$$

and, as we said earlier, there is a number $r$ such that the rank of (3) is equal to $r$ for all $t>r$ and is equal to $t$ for all $t \leq r$. Thus, the determinant of (2) is 0 for all $t>r$ and is nonzero for all $t \leq r$, leading to the following result.

Theorem 1. Let $w_{0}, w_{1}, \ldots, w_{2 t-2}$ be the number of closed walks in $G$ of length $0,1, \ldots, 2 t-2$ that start and end at vertex $v$. Then there exists a number $r$ such that the determinant of

$$
\left(\begin{array}{ccccc}
w_{0} & w_{1} & w_{2} & \cdots & w_{t-1} \\
w_{1} & w_{2} & w_{3} & . & w_{t} \\
w_{2} & w_{3} & . \cdot & . \cdot & w_{t+1} \\
\vdots & . \cdot & . \cdot & . \cdot & \vdots \\
w_{t-1} & w_{t} & w_{t+1} & \cdots & w_{2 t-2}
\end{array}\right)
$$

is zero for all $t>r$ and is nonzero for all $t \leq r$. Moreover, the rank of $\mathbf{W}_{v}$ is $r$.

Note also that, referring to (1), each of the upper $p \times p$ submatrices of $\mathbf{H}_{v}$ are invertible. This may be deduced from the fact that $\mathbf{H}_{v}$ is positive definite. Alternatively, any upper $p \times p$ submatrix of the $r \times r$ matrix $\mathbf{H}_{v}$ is equal to $\mathbf{W}_{v p}^{\top} \mathbf{W}_{v p}$, where $\mathbf{W}_{v p}$ is $\mathbf{W}_{v}$ restricted to its first $p$ columns, and this matrix has a full rank by Theorem 1.

\section{Companion Matrices and Eigenvalues}

The rank of a walk matrix $\mathbf{W}_{\mathbf{b}}$ may also be evaluated by finding the number of eigenvalues of $G$ having an associated eigenvector that is not orthogonal to $\mathbf{b}[17,18]$. In our case, since the walk matrices under discussion have $\mathbf{b}=\mathbf{e}_{v}$, where $v=1,2, \ldots, n$, we can equivalently say the following. 
Theorem 2 ([17]). The rank of $\mathbf{W}_{v}$ is the number of eigenvalues of $G$ with an associated eigenvector having a nonzero vth entry.

The significance of zero and nonzero entries in eigenvectors has found applications in control theory $[17,18,21]$ and in molecular conduction $[2,22,23]$.

For any walk matrix $\mathbf{W}_{\mathbf{b}}$, its companion matrix $\mathbf{C}_{\mathbf{b}}$ is the matrix satisfying

$$
A W_{b}=W_{b} C_{b}
$$

For any vertex $v \in \mathcal{V}(G)$, we denote the companion matrix of $\mathbf{W}_{v}$ by $\mathbf{C}_{v}$. As we shall see soon, $\mathbf{C}_{v}$ may be different for each $\mathbf{W}_{v}$. Indeed, if $\mathbf{W}_{v}$ is an $n \times r$ matrix, then $\mathbf{C}_{v}$ is the $r \times r$ matrix $\left(\begin{array}{lllll}\mathbf{e}_{2} & \mathbf{e}_{3} & \cdots & \mathbf{e}_{r} & \mathbf{c}_{v}\end{array}\right)$ for an appropriate column vector $\mathbf{c}_{v}$. As can be seen if we evaluate the determinant of $x \mathbf{I}-\mathbf{C}_{v}$ using the Laplace determinant expansion along the last column, the characteristic polynomial of $\mathbf{C}_{v}$ is

$$
\phi_{v}(x)=x^{r}-c_{r-1}^{(v)} x^{r-1}-c_{r-2}^{(v)} x^{r-2}-\cdots-c_{1}^{(v)} x-c_{0}^{(v)}
$$

where the vector $\left(\begin{array}{lllll}c_{0}^{(v)} & c_{1}^{(v)} & \cdots & c_{r-2}^{(v)} & c_{r-1}^{(v)}\end{array}\right)$ is $\mathbf{c}_{v}^{\top}$. It is known that the coefficients of $\phi_{v}(x)$ are integers [16].

Moreover, and more importantly, $\phi_{v}(x)$ divides the characteristic polynomial of $G[15,19]$. Here, we provide an alternative proof of this result by stating the roots of $\phi_{v}(x)$.

Theorem 3. Let $\mathbf{C}_{v}$ be the companion matrix of $\mathbf{W}_{v}$ such that $\mathbf{A} \mathbf{W}_{v}=\mathbf{W}_{v} \mathbf{C}_{v}$ and let $\phi_{v}(x)$ be the characteristic polynomial of $\mathbf{C}_{v}$. The roots of $\phi_{v}(x)$ are the eigenvalues of $G$ whose eigenspaces contain an eigenvector with a nonzero entry in its vth position.

Proof. Let $\lambda$ be any eigenvalue of $\mathbf{A}$ with associated eigenvector $\mathbf{x}$. We take the transpose on both sides of the relation $\mathbf{A} \mathbf{W}_{v}=\mathbf{W}_{v} \mathbf{C}_{v}$ to obtain $\mathbf{C}_{v}^{\top} \mathbf{W}_{v}^{\top}=\mathbf{W}_{v}^{\top} \mathbf{A}$. By postmultiplying both sides of this equality by $\mathbf{x}$, we get

$$
\mathbf{C}_{v}\left(\mathbf{W}_{v}^{\top} \mathbf{x}\right)=\lambda\left(\mathbf{W}_{v}^{\top} \mathbf{x}\right)
$$

But

$$
\mathbf{W}_{v}^{\top} \mathbf{x}=\left(\begin{array}{c}
\mathbf{e}_{v}^{\top} \\
\mathbf{e}_{v}^{\top} \mathbf{A} \\
\mathbf{e}_{v}^{\top} \mathbf{A}^{2} \\
\vdots \\
\mathbf{e}_{v}^{\top} \mathbf{A}^{r-1}
\end{array}\right) \mathbf{x}=\left(\begin{array}{c}
\mathbf{e}_{v}^{\top} \mathbf{x} \\
\lambda \mathbf{e}_{v}^{\top} \mathbf{x} \\
\lambda^{2} \mathbf{e}_{v}^{\top} \mathbf{x} \\
\vdots \\
\lambda^{r-1} \mathbf{e}_{v}^{\top} \mathbf{x}
\end{array}\right)=\left(\mathbf{e}_{v}^{\top} \mathbf{x}\right)\left(\begin{array}{c}
1 \\
\lambda \\
\lambda^{2} \\
\vdots \\
\lambda^{r-1}
\end{array}\right)
$$

Hence $\mathbf{W}_{v}^{\top} \mathbf{x}=\mathbf{0}$ if and only if $\mathbf{x}$ has a zero entry in its $v$ th position. Thus, referring to (4) and (5), whenever an eigenvector $\mathbf{x}$ associated with $\lambda$ in $\mathbf{A}$ has a nonzero entry in its $v$ th position, the (nonzero) vector $\left(\begin{array}{lllll}1 & \lambda & \lambda^{2} & \cdots & \lambda^{r-1}\end{array}\right)^{\top}$ would be an eigenvector associated with $\lambda$ in $\mathbf{C}_{v}^{\top}$. Since the choice of the eigenvalue $\lambda$ in $\mathbf{A}$ was arbitrary, we have proved the result.

By combining Theorems 2 and 3 together, the following corollary is determined immediately.

Corollary 1. The rank of $\mathbf{W}_{v}$ is equal to the degree of $\phi_{v}(x)$.

The following theorem conveys the fact that if $r$ is the rank of $\mathbf{W}_{v}$, then the $r \times r$ companion matrix $\mathbf{C}_{v}$ may be found solely from walk enumerations of closed walks of length up to $2 r-1$ that start and end at vertex $v$ in $G$. The argument of Theorem 4 below is based on the result in ([24] p. 43) stating that the last column of $\mathbf{C}_{v}$ is equal to $\mathbf{H}_{v}^{-1} \mathbf{W}_{v}^{\top} \mathbf{A}^{r} \mathbf{e}_{v}$. 
Theorem 4. The $r \times r$ companion matrix of $\mathbf{W}_{v}$ is the matrix $\left(\begin{array}{llllll}\mathbf{e}_{2} & \mathbf{e}_{3} & \cdots & \mathbf{e}_{r} & \mathbf{c}_{v}\end{array}\right)$, where

$$
\mathbf{c}_{v}=\left(\begin{array}{ccccc}
w_{0} & w_{1} & w_{2} & \cdots & w_{r-1} \\
w_{1} & w_{2} & w_{3} & . \cdot & w_{r} \\
w_{2} & w_{3} & . \cdot & . \cdot & w_{r+1} \\
\vdots & . \cdot & . \cdot & . \cdot & \vdots \\
w_{r-1} & w_{r} & w_{r+1} & \cdots & w_{2 r-2}
\end{array}\right)^{-1}\left(\begin{array}{c}
w_{r} \\
w_{r+1} \\
w_{r+2} \\
\vdots \\
w_{2 r-1}
\end{array}\right)
$$

and $w_{0}, w_{1}, \ldots, w_{2 r-1}$ are the number of closed walks of length $0,1, \ldots, 2 r-1$ that start and end at vertex $v$.

Proof. By comparing the last columns of the equality $\mathbf{A} \mathbf{W}_{v}=\mathbf{W}_{v} \mathbf{C}_{v}$, we obtain the relationship $\mathbf{A}^{r} \mathbf{e}_{v}=\mathbf{W}_{v} \mathbf{c}_{v}$. Thus, $\mathbf{W}_{v}^{\top} \mathbf{A}^{r} \mathbf{e}_{v}=\mathbf{H}_{v} \mathbf{c}_{v}$, which may be rewritten as

$$
\left(\begin{array}{c}
\mathbf{e}_{v}^{\top} \\
\mathbf{e}_{v}^{\top} \mathbf{A} \\
\mathbf{e}_{v}^{\top} \mathbf{A}^{2} \\
\vdots \\
\mathbf{e}_{v}^{\top} \mathbf{A}^{r-1}
\end{array}\right)\left(\mathbf{A}^{r} \mathbf{e}_{v}\right)=\mathbf{H}_{v} \mathbf{c}_{v}
$$

or as

$$
\mathbf{H}_{v} \mathbf{c}_{v}=\left(\begin{array}{lllll}
w_{r} & w_{r+1} & w_{r+2} & \cdots & w_{2 r-1}
\end{array}\right)^{\top} .
$$

The proof is complete since $\mathbf{H}_{v}$ is invertible.

The reference ([15] Theorem 2.3) contains a result akin to Theorem 4 that instead extracts each entry of $\mathbf{c}_{v}$ one at a time from the walk enumerations $w_{0}, \ldots, w_{2 r-1}$.

We emphasize what we have accomplished in Theorem 4 by presenting the following corollary.

Corollary 2. For any vertex $v$, let the characteristic polynomial of $\mathbf{C}_{v}$ be $\phi_{v}(x)$. If $\phi_{v}(x)$ is of degree $r$, then $\phi_{v}(x)$ is deducible from $w_{0}(v), w_{1}(v), \ldots, w_{2 r-1}(v)$.

\section{Eigenvalues of Vertex Deleted Subgraphs}

Recall that the vertex deleted subgraph $G-v$ of $G$ is obtained from $G$ by removing vertex $v$ and all edges incident to it. The common eigenvalues of $G-v$ and $G$ are also able to tell us the rank of $\mathbf{W}_{v}$. The reason for this is presented in the proof of Theorem 7 below.

Before proving Theorem 7, we first prove the following result that allows us to deduce the common eigenvalues of $G$ and $G-v$ from the eigenspaces of $G$. The result of Theorem 5 is required for the proof of Theorem 7 .

Theorem 5 ([17]). Let $\lambda$ be an eigenvalue of $G$ with eigenspace $E(\lambda)$. Then $\lambda$ is also an eigenvalue of $G-v$ if and only if $E(\lambda)$ contains an eigenvector whose vth entry is zero.

Proof. Let the adjacency matrices of $G$ and $G-v$ be $\mathbf{A}$ and $\mathbf{A}_{v}$, respectively. After reordering the vertices of $G$ if necessary, $\mathbf{A}$ may be partitioned into the block matrix

$$
\mathbf{A}=\left(\begin{array}{cc}
\mathbf{A}_{v} & \mathbf{s} \\
\mathbf{s}^{\top} & 0
\end{array}\right)
$$

for some indicator vector $\mathbf{s}$ of the adjacencies of vertex $v$. We also have $\mathbf{A x}=\lambda \mathbf{x}$ for some eigenvector $\mathbf{x}$ in $E(\lambda)$.

Suppose $\lambda$ is an eigenvalue of both $G$ and of $G-v$. We prove that $E(\lambda)$ contains an eigenvector with a zero in its $v$ th position. Let $\mathbf{A}_{v} \mathbf{y}=\lambda \mathbf{y}$ for some eigenvector $\mathbf{y}$ pertaining 
to the eigenvalue $\lambda$ in the eigenspace of $G-v$. Let $\mathbf{x}$ be partitioned into $(\mathbf{z} k)^{\top}$, where $\mathbf{z}$ is $(n-1)$-dimensional. Then $\mathbf{A x}=\lambda \mathbf{x}$ may be rewritten as

$$
\left(\begin{array}{cc}
\mathbf{A}_{v} & \mathbf{s} \\
\mathbf{s}^{\top} & 0
\end{array}\right)\left(\begin{array}{l}
\mathbf{z} \\
k
\end{array}\right)=\left(\begin{array}{l}
\lambda \mathbf{z} \\
\lambda k
\end{array}\right)
$$

from which we obtain, in particular, the relation

$$
\mathbf{A}_{v} \mathbf{z}+k \mathbf{s}=\lambda \mathbf{z}
$$

By premultiplying both sides of (6) by $\mathbf{y}^{\top}$, we obtain

$$
\mathbf{y}^{\top} \mathbf{A}_{v} \mathbf{z}+k \mathbf{y}^{\top} \mathbf{s}=\lambda \mathbf{y}^{\top} \mathbf{z}
$$

which simplifies to

$$
k\left(\mathbf{y}^{\top} \mathbf{s}\right)=0 .
$$

Hence, either $k=0$ or $\mathbf{y}^{\top} \mathbf{s}=0$. If $k=0$, then we have proved the result, since then $\mathbf{x}=\left(\begin{array}{ll}\mathbf{z} & 0\end{array}\right)^{\top}$. If $\mathbf{y}^{\top} \mathbf{s}=0$, then we claim that $\left(\begin{array}{ll}\mathbf{y} & 0\end{array}\right)^{\top} \in E(\lambda) ;$ indeed

$$
\left(\begin{array}{cc}
\mathbf{A}_{v} & \mathbf{s} \\
\mathbf{s}^{\top} & 0
\end{array}\right)\left(\begin{array}{l}
\mathbf{y} \\
0
\end{array}\right)=\left(\begin{array}{c}
\mathbf{A}_{v} \mathbf{y} \\
\mathbf{s}^{\top} \mathbf{y}
\end{array}\right)=\lambda\left(\begin{array}{l}
\mathbf{y} \\
0
\end{array}\right) .
$$

Thus, either way, $\lambda$ has an eigenvector in $E(\lambda)$ with a zero entry in its $v$ th position, proving sufficiency.

Conversely, suppose $\lambda$ is an eigenvalue of $G$ with an associated eigenvector having a zero in its $v$ th position. We prove that $\lambda$ is also an eigenvalue of $G-v$. Let $\mathbf{x}$ be partitioned into $\left(\begin{array}{ll}\mathbf{z} & 0\end{array}\right)^{\top}$. Since $\mathbf{A x}=\lambda \mathbf{x}$,

$$
\left(\begin{array}{cc}
\mathbf{A}_{v} & \mathbf{s} \\
\mathbf{s}^{\top} & 0
\end{array}\right)\left(\begin{array}{l}
\mathbf{z} \\
0
\end{array}\right)=\left(\begin{array}{c}
\mathbf{A}_{v} \mathbf{z} \\
\mathbf{s}^{\top} \mathbf{z}
\end{array}\right)=\left(\begin{array}{c}
\lambda \mathbf{z} \\
0
\end{array}\right) .
$$

Thus, in particular, $\mathbf{A}_{v} \mathbf{z}=\lambda \mathbf{z}$, proving that $\lambda$ is also an eigenvalue of $G-v$.

We arrive at yet another way of determining the rank of $\mathbf{W}_{v}$, which is illustrated in Theorem 7. The proof of this result uses the so-called Interlacing theorem (see [25], for instance), presented below for the case of eigenvalues of graphs.

Theorem 6 (Interlacing theorem [25]). Let $G$ be a graph on $n$ vertices having the $n$ eigenvalues $\lambda_{1} \geq \lambda_{2} \geq \cdots \geq \lambda_{n}$ and let the vertex deleted subgraph $G-v$ have the $n-1$ eigenvalues $\mu_{1} \geq \mu_{2} \geq \cdots \geq \mu_{n-1}$. Then

$$
\lambda_{1} \geq \mu_{1} \geq \lambda_{2} \geq \mu_{2} \geq \cdots \geq \lambda_{n-1} \geq \mu_{n-1} \geq \lambda_{n}
$$

that is, the eigenvalues of $G$ and $G-v$ interlace.

As an immediate corollary of Theorem 6, the multiplicity of any eigenvalue $\lambda$ of a graph $G$ must be at most one more than the multiplicity of the same eigenvalue $\lambda$ in the vertex deleted subgraph $G-v$. The statement of Theorem 7 below concerns eigenvalues of $G$ whose multiplicities are exactly one more than those for the same eigenvalues in $G-v$. In its proof argument, an eigenvalue is assumed to have multiplicity zero if it is not an eigenvalue of its adjacency matrix.

Theorem 7. Let $\lambda$ be an eigenvalue of $G$ whose multiplicity is one more than the multiplicity of $\lambda$ in the vertex deleted subgraph $G-v$. The rank of $\mathbf{W}_{v}$ is the number of all such distinct eigenvalues of $G$. 
Proof. Let $\lambda$ have multiplicity $q$ in $G$ and let $\left\{\mathbf{x}_{1}, \ldots, \mathbf{x}_{q}\right\}$ be an eigenbasis for $\lambda$ in $G$. We assume that all of the eigenvectors $x_{2}, \ldots, \mathbf{x}_{q}$ have their $v$ th entry equal to zero. If this is not the case, so that, without loss of generality, both $\mathbf{x}_{1}$ and $\mathbf{x}_{j}$ have a nonzero $i$ th entry, then replace $\mathbf{x}_{j}$ by $\left(\mathbf{e}_{v}^{\top} \mathbf{x}_{j}\right) \mathbf{x}_{1}-\left(\mathbf{e}_{v}^{\top} \mathbf{x}_{1}\right) \mathbf{x}_{j}$.

By the Interlacing theorem (Theorem 6), the multiplicity of $\lambda$ in $G-v$ cannot be less than $q-1$. Let $\mathbf{z}-v$ be the vector $\mathbf{z}$ without its $v$ th entry. By the argument presented in the proof of Theorem 5 , all of the eigenvectors $\mathbf{x}_{2}-v, \ldots, \mathbf{x}_{q}-v$ will also be eigenvectors of $\lambda$ in $G-v$, and clearly these $q-1$ eigenvectors are linearly independent. If $\mathbf{x}_{1}$ also has its $v$ th entry equal to zero, then $\mathbf{x}_{1}-v$ will also be an eigenvector of $\lambda$ in $G-v$ that is linearly independent of $\mathbf{x}_{2}-v, \ldots, \mathbf{x}_{q}-v$, so that the multiplicity of $\lambda$ in $G-v$ would be at least $q$. However, then that would mean that any linear combination of the vectors in the eigenbasis of $\lambda$ in $G$ would have a zero in its vth position, which, by Theorem 2, would lead to $\lambda$ not contributing to the rank of $\mathbf{W}_{v}$. Thus, the eigenvalues that contribute to the rank of $\mathbf{W}_{v}$ are precisely those whose multiplicity is one more than that of the same eigenvalue in $G-v$, as required.

A vertex $v$ whose removal from $G$ reduces the multiplicity of an eigenvalue $\lambda$ in $G$ by one is called a $\lambda$-core vertex [26] or a downer vertex [27]. Thus, Theorem 7 may be restated as follows:

Theorem 8. The rank of $\mathbf{W}_{v}$ is the number of distinct eigenvalues of $G$ for which $v$ is a core/downer vertex.

The eigenvalues of $G$ described in the statement of Theorem 7 or Theorem 8 are precisely the roots of $\phi_{v}(x)$. The following theorem proves this, and more.

Theorem 9. Let $G$ be a graph and let $v$ be any of its vertices. The $r$ distinct roots of $\phi_{v}(x)$ are the eigenvalues of $G$ for which $v$ is a core/downer vertex. Moreover, the $n-r$ remaining eigenvalues of $G$ (including multiplicities) are also eigenvalues of $G-v$.

Proof. Let $\lambda$ be any root of $\phi_{v}(x)$. By Theorem $3, \lambda$ is an eigenvalue of $G$ whose eigenspace $E(\lambda)$ contains an eigenvector with a nonzero number in its $v$ th entry. As described in the first paragraph of the proof of Theorem 7, a basis $\left\{\mathbf{x}_{1}, \mathbf{x}_{2}, \ldots, \mathbf{x}_{q}\right\}$ for $E(\lambda)$ can be chosen such that only $\mathbf{x}_{1}$ has a nonzero entry in its $v$ th position. By the argument in the second paragraph of the proof of Theorem 7 , the multiplicity of $\lambda$ in $G-v$ must be $q-1$, proving the first part of the theorem statement.

Now suppose $\mu$ is an eigenvalue of $G$ that is not a root of $\phi_{v}(x)$. Then by Theorem 3, $E(\mu)$ contains only vectors whose $v$ th entry is zero. Thus, any eigenbasis for $E(\mu)$ must be made up of such vectors as well. By again applying the argument used in Theorem 7 , the multiplicity of $\mu$ in $G-v$ must be at least equal to that of $\mu$ in $G$, which proves the second part of the theorem statement.

We end this section by summarizing the various ways described in this paper to obtain the rank of $\mathbf{W}_{v}$.

Theorem 10. The following are all equal to the rank of $\mathbf{W}_{v}$ :

- One less than the order of the smallest singular matrix whose skew diagonal entries are constant and equal to $w_{0}(v), w_{1}(v), w_{2}(v), \ldots$ from left to right (Theorem 1);

- $\quad$ The degree of $\phi_{v}(x)$ (Corollary 1);

- $\quad$ The number of distinct eigenvalues of $G$ with an associated eigenvector having a nonzero entry in its vth position (Theorem 2);

- $\quad$ The number of distinct eigenvalues of $G$ for which $v$ is a core/downer vertex (Theorem 7 ). 


\section{Reconstruction}

Let $\mathscr{P}(G)$ be the polynomial deck $\{\phi(G-1, x), \phi(G-2, x), \ldots, \phi(G-n, x)\}$ containing the (unordered) characteristic polynomials of all vertex deleted subgraphs of $G$. As is well-known, the derivative of the characteristic polynomial of $G$ may be reconstructed from $\mathscr{P}(G)$ as in the following result.

Theorem 11 ([28,29]). The derivative of the characteristic polynomial of $G$ is equal to $\phi(G-1, x)+\phi(G-2, x)+\cdots+\phi(G-n, x)$.

Thus, all the coefficients of $\phi(G, x)$ may be reconstructed from $\mathscr{P}(G)$, except possibly the constant one. Furthermore, if one of the members of $\mathscr{P}(G)$ has a multiple root, then by Theorem 6, this root must also be a root of $\phi(G, x)$, and $\phi(G, x)$ is reconstructed immediately.

The number of closed walks of length $0,1, \ldots, n-1$ starting and ending at any vertex of $G$ are also reconstructible from $\mathscr{P}(G)$. This was proved in [1]. Below, we slightly elaborate on the proof that is provided there.

Theorem $12\left([1]\right.$ Theorem 2). For all $v \in \mathcal{V}(G)$, the walks $w_{0}(v), \ldots, w_{n-1}(v)$ may be reconstructed from $\mathscr{P}(G)$.

Proof. From ([29] p. 34), the formal power series $\sum_{j=0}^{\infty} w_{j}(v) x^{j}$ may be generated by the following generating function:

$$
\frac{\phi\left(G-v, x^{-1}\right)}{x \phi\left(G, x^{-1}\right)} .
$$

Note that the rational function (7) may be rewritten as

$$
\frac{\phi^{\star}(G-v, x)}{\phi^{\star}(G, x)}
$$

where the notation $p^{\star}(x)$ represents the reflected polynomial of $p(x)$, that is, the expression $x^{n} p\left(x^{-1}\right)$, the polynomial $p(x)$ with its coefficients in reverse order [30,31]. Hence

$$
\phi^{\star}(G, x)\left(w_{0}(v)+w_{1}(v) x+w_{2}(v) x^{2}+\cdots\right)=\phi^{\star}(G-v, x) .
$$

Since the only unknown coefficient of $\phi^{\star}(G, x)$ is the leading one, the numbers $w_{0}(v), \ldots$, $w_{n-1}(v)$ may be discovered by expanding the left hand side of (8) and comparing coefficients.

We have finally conveyed all the results we require in order to prove the main result of this paper, which is presented in Theorem 13 underneath. It is assumed that, when counting the roots of any polynomial mentioned in the statement of Theorem 13, multiple roots are counted as many times as their multiplicity.

Theorem 13. Let $\mathscr{P}(G)$ be the polynomial deck $\{\phi(G-1, x), \phi(G-2, x), \ldots, \phi(G-n, x)\}$ containing the characteristic polynomials of all vertex deleted subgraphs of $G$. Then $\mathscr{P}(G)$ determines whether or not $\phi(G, x)$ has more than half of its $n$ roots included among the $n-1$ roots of one of its characteristic polynomials. When this is the case, $\phi(G, x)$ is reconstructible from $\mathscr{P}(G)$. 
Proof. For each vertex $v$, we first obtain the walk enumerations $w_{0}(v), \ldots, w_{n-1}(v)$ using Theorem 12. Moreover, for each $v$, we calculate the determinant

$$
\left|\begin{array}{ccccc}
w_{0}(v) & w_{1}(v) & w_{2}(v) & \cdots & w_{t-1}(v) \\
w_{1}(v) & w_{2}(v) & w_{3}(v) & . & w_{t}(v) \\
w_{2}(v) & w_{3}(v) & . & . & w_{t+1}(v) \\
\vdots & . & . & . & \vdots \\
w_{t-1}(v) & w_{t}(v) & w_{t+1}(v) & \cdots & w_{2 t-2}(v)
\end{array}\right|
$$

where $2 t-2$ is either $n-1$ or $n-2$, depending on the parity of $n$. If one of these determinants is zero for some particular vertex $v$, then by Theorem 1 , the rank of $\mathbf{W}_{v}, r$, is the order of the largest nonsingular principal upper submatrix of this determinant, which is less than $t$. Indeed, if this happens, then when $n$ is odd, $t=\frac{n+1}{2}$, so $r<\frac{n+1}{2}<\frac{n}{2}$, and when $n$ is even, $t=\frac{n}{2}$, so $r<\frac{n}{2}$. Either way, we infer that $r$ is less than half $n$ whenever (9) is zero for some vertex $v$.

For this particular vertex $v$, we now use Theorem 4 to obtain the companion characteristic polynomial $\phi_{v}(x)$ from $w_{0}, \ldots, w_{2 r-1}$. By Theorem 3 , the $r$ roots of $\phi_{v}(x)$ are $r$ of the $n$ roots of $\phi(G, x)$. Moreover, by Theorem 9 , the remaining $n-r$ roots of $\phi(G, x)$ will also be eigenvalues of $G-v$. However, since $r\left\langle\frac{n}{2}, n-r\right\rangle \frac{n}{2}$. We have determined, therefore, that when the determinant (9) is zero for some vertex $v$, then $G$ must share more than half of its eigenvalues (if we include repetitions) with $G-v$. Since (9) may be found from $\mathscr{P}(G)$, the fact that $G$ shares more than half of its eigenvalues with $G-v$ is recognizable from $\mathscr{P}(G)$.

To reconstruct $\phi(G, x)$, we use the fact that $\phi_{v}(x)$ divides $\phi(G, x)$. By Theorem 11, all the coefficients of $\phi(G, x)$ bar the constant one $K$ are reconstructible from $\mathscr{P}(G)$. Since $\phi_{v}(x)$ is available, we reconstruct $\phi(G, x)$ by, for instance, performing the polynomial division $\frac{\phi(G, x)}{\phi_{v}(x)}$ and solving for $K$.

Remark 1. Focusing on the last sentence of the proof of Theorem 13 for a moment, the eigenvalues shared by $G$ and $G-v$ would be the $n-r$ roots of the polynomial $\frac{\phi(G, x)}{\phi_{v}(x)}$.

\section{Examples}

We now illustrate the techniques in the proof of Theorem 13 by successfully reconstructing the characteristic polynomials of the following two example polynomial decks.

\subsection{First Example}

The polynomial deck contains the seven polynomials

$$
\begin{aligned}
& \phi(G-1, x)=x^{6}-7 x^{4}+9 x^{2}-4 x \\
& \phi(G-2, x)=x^{6}-7 x^{4}+9 x^{2}-4 x \\
& \phi(G-3, x)=x^{6}-6 x^{4}-2 x^{3}+6 x^{2}+2 x-1, \\
& \phi(G-4, x)=x^{6}-6 x^{4}-2 x^{3}+6 x^{2}+2 x-1, \\
& \phi(G-5, x)=x^{6}-8 x^{4}-4 x^{3}+6 x^{2}+2 x-1, \\
& \phi(G-6, x)=x^{6}-8 x^{4}-4 x^{3}+6 x^{2}+2 x-1, \\
& \phi(G-7, x)=x^{6}-8 x^{4}-4 x^{3}+12 x^{2}+4 x-5 .
\end{aligned}
$$

All of $\phi(G-1, x), \ldots, \phi(G-7, x)$ have six distinct roots. The sum of these seven polynomials is $7 x^{6}-50 x^{4}-16 x^{3}+54 x^{2}+4 x-9$; thus, by integration, $\phi(G, x)$ is the polynomial $x^{7}-10 x^{5}-4 x^{4}+18 x^{3}+2 x^{2}-9 x+K$, according to Theorem 11 . Thus, as soon as we discover the value of $K$, the reconstruction of $\phi(G, x)$ would be complete. 
The roots of $\phi(G-7, x)$ are $2.79129,1,0.61803,-1,-1.61803,-1.79129$. We show that more than half of the seven roots of $\phi(G, x)$ (in this case, more than three) are among these six roots.

We find $w_{0}, w_{1}, \ldots, w_{6}$, the number of closed walks of length $0,1, \ldots, 6$ starting and ending at vertex 7 in G. By using Theorem 12, particularly (8):

$$
\begin{aligned}
\left(1-10 x^{2}-4 x^{3}+18 x^{4}+2 x^{5}-9 x^{6}+K x^{7}\right)\left(w_{0}+\right. & \left.w_{1} x+w_{2} x^{2}+\cdots\right) \\
& =1-8 x^{2}-4 x^{3}+12 x^{4}+4 x^{5}-5 x^{6}
\end{aligned}
$$

whence we obtain $w_{0}=1, w_{1}=0, w_{2}=2, w_{3}=0, w_{4}=14, w_{5}=10, w_{6}=108$. In line with Theorem 1, we calculate the determinant

$$
\left|\begin{array}{cccc}
1 & 0 & 2 & 0 \\
0 & 2 & 0 & 14 \\
2 & 0 & 14 & 10 \\
0 & 14 & 10 & 108
\end{array}\right|
$$

Since this determinant is zero, $G$ must share at least four eigenvalues with $G-7$, by Theorem 13. The upper principal minor

$$
\left|\begin{array}{ccc}
1 & 0 & 2 \\
0 & 2 & 0 \\
2 & 0 & 14
\end{array}\right|
$$

is nonzero, so the rank of $\mathbf{W}_{7}$ is three, by Theorem 1 . Hence $G$ shares exactly $7-3=4$ eigenvalues with $G-7$. However, we do not know which are these four eigenvalues yet.

In accordance with Theorem 4 , we evaluate the matrix product

$$
\left(\begin{array}{ccc}
1 & 0 & 2 \\
0 & 2 & 0 \\
2 & 0 & 14
\end{array}\right)^{-1}\left(\begin{array}{c}
0 \\
14 \\
10
\end{array}\right)
$$

obtaining $\left(\begin{array}{lll}-2 & 7 & 1\end{array}\right)^{\top}$. Thus, the companion polynomial of $\mathbf{W}_{7}, \phi_{7}(x)$, is equal to $x^{3}-$ $x^{2}-7 x+2$. This polynomial divides $\phi(G, x)$, by Theorem 3. By performing polynomial division of $\phi(G, x)$ by $\phi_{7}(x)$, say, we deduce that $K=2$.

We have thus successfully reconstructed $\phi(G, x)$ as being $x^{7}-10 x^{5}-4 x^{4}+18 x^{3}+$ $2 x^{2}-9 x+2$. Note that its roots are $3.06871,1,0.61803,0.27775,-1,-1.61803,-2.34646$. The three roots $3.06871,0.27775,-2.34646$ are those of $\phi_{7}(x)$, while the four remaining roots $1,0.61803,-1,-1.61803$ are shared with those of $\phi(G-7, x)$, confirming that $G$ does share four of its eigenvalues with $G-7$.

\subsection{Second Example}

The polynomial deck contains the eight polynomials

$$
\begin{aligned}
\phi(G-1, x) & =x^{7}-8 x^{5}-2 x^{4}+16 x^{3}+2 x^{2}-9 x+2, \\
\phi(G-2, x) & =x^{7}-8 x^{5}-2 x^{4}+16 x^{3}+2 x^{2}-9 x+2, \\
\phi(G-3, x) & =x^{7}-10 x^{5}-8 x^{4}+16 x^{3}+12 x^{2}-7 x-2, \\
\phi(G-4, x) & =x^{7}-10 x^{5}-8 x^{4}+16 x^{3}+12 x^{2}-7 x-2, \\
\phi(G-5, x) & =x^{7}-9 x^{5}-6 x^{4}+15 x^{3}+12 x^{2}-3 x-2, \\
\phi(G-6, x) & =x^{7}-9 x^{5}-6 x^{4}+15 x^{3}+12 x^{2}-3 x-2, \\
\phi(G-7, x) & =x^{7}-10 x^{5}-6 x^{4}+21 x^{3}+14 x^{2}-12 x-8, \\
\phi(G-8, x) & =x^{7}-8 x^{5}-2 x^{4}+17 x^{3}+6 x^{2}-10 x-4 .
\end{aligned}
$$


All of $\phi(G-1, x), \ldots, \phi(G-8, x)$ have seven distinct roots. The sum of these eight polynomials is $8 x^{7}-72 x^{5}-40 x^{4}+132 x^{3}+72 x^{2}-60 x-16$; thus, by Theorem $11, \phi(G, x)=$ $x^{8}-12 x^{6}-8 x^{5}+33 x^{4}+24 x^{3}-30 x^{2}-16 x+K$, after integrating this sum with respect to $x$. As before, we now endeavour to determine the value of $K$.

The roots of $\phi(G-8, x)$ are $2.41421,1.41421,1,-0.41421,-1,-1.41421,-2$. We show that more than half-that is, more than four-of the eight roots of $\phi(G, x)$ are among these seven roots.

The number of closed walks of length $0,1, \ldots, 7$ starting and ending at vertex 8 in $G$ may again be obtained by comparing the coefficients of both sides of two appropriate formal power series in agreement with (8):

$$
\begin{array}{r}
\left(1-12 x^{2}-8 x^{3}+33 x^{4}+24 x^{5}-30 x^{6}-16 x^{7}+K x^{8}\right)\left(w_{0}+w_{1} x+w_{2} x^{2}+\cdots\right) \\
=1-8 x^{2}-2 x^{3}+17 x^{4}+6 x^{5}-10 x^{6}-4 x^{7} .
\end{array}
$$

We obtain the values $w_{0}=1, w_{1}=0, w_{2}=4, w_{3}=6, w_{4}=32, w_{5}=86, w_{6}=320$, $w_{7}=1006$. By showing that the determinant

$$
\left|\begin{array}{cccc}
1 & 0 & 4 & 6 \\
0 & 4 & 6 & 32 \\
4 & 6 & 32 & 86 \\
6 & 32 & 86 & 320
\end{array}\right|
$$

is zero (Theorem 1) we deduce that $G$ must share at least five eigenvalues with $G-8$ (Theorem 13). Note that $w_{7}$ is not used here, because the number of vertices of $G$ is even. The upper principal minor

$$
\left|\begin{array}{ccc}
1 & 0 & 4 \\
0 & 4 & 6 \\
4 & 6 & 32
\end{array}\right|
$$

is nonzero-thus, the rank of $\mathbf{W}_{8}$ is three. This means that $G$ shares exactly $8-3=5$ eigenvalues with $G-8$; however, these five eigenvalues are unknown, for now.

We now discover $\phi_{8}(x)$, the companion polynomial of $\mathbf{W}_{8}$. To this end, we work out the product

$$
\left(\begin{array}{ccc}
1 & 0 & 4 \\
0 & 4 & 6 \\
4 & 6 & 32
\end{array}\right)^{-1}\left(\begin{array}{c}
6 \\
32 \\
86
\end{array}\right)
$$

obtaining $\left(\begin{array}{lll}-2 & 5 & 2\end{array}\right)^{\top}$. Hence, in line with Theorem 4, the companion polynomial of $\mathbf{W}_{8}$ is $\phi_{8}(x)=x^{3}-2 x^{2}-5 x+2$. Since $\phi_{8}(x)$ divides $\phi(G, x)$ (Theorem 3), we deduce that $K=8$, and hence $\phi(G, x)=x^{8}-12 x^{6}-8 x^{5}+33 x^{4}+24 x^{3}-30 x^{2}-16 x+8$.

The roots of $\phi(G, x)$ are $3.32340,1.41421,1,0.35793,-1,-1.41421,-1.68133,-2$. The three roots $3.32340,0.35793,-1.61833$ are those of $\phi_{8}(x)$, while the five remaining roots $1.41421,1,-1,-1.41421,-2$ are shared with those of $\phi(G-8, x)$.

\section{Disconnected Graphs}

Our main result, Theorem 13, reconstructs $\phi(G, x)$ from $\mathscr{P}(G)$ by obtaining a companion polynomial $\phi_{v}(x)$ after deducing that the degree of $\phi_{v}(x)$ is smaller than half of $|\mathcal{V}(G)|$. This companion polynomial, having integer coefficients, is a factor of $\phi(G, x)$.

It is known that if $G$ is a connected graph, then the largest eigenvalue of $\phi(G, x)$ is always a root of $\phi_{v}(x)$ for any $v \in \mathcal{V}(G)$ [16]. Hence, Theorem 13 cannot be used for connected graphs whose largest eigenvalue has a minimal polynomial whose degree is at least half of $|\mathcal{V}(G)|$. This includes connected graphs whose characteristic polynomial is irreducible over $\mathbb{Q}$.

Note, however, that if $G$ is a disconnected graph having two components $G_{1}$ and $G_{2}$, then $\phi(G, x)=\phi\left(G_{1}, x\right) \phi\left(G_{2}, x\right)$. If $\left|\mathcal{V}\left(G_{1}\right)\right|<\left|\mathcal{V}\left(G_{2}\right)\right|$, then Theorem 13 is always able 
to reconstruct $\phi(G, x)$ from $\mathscr{P}(G)$. If $G$ has more than two components or $|\mathcal{V}(G)|$ is odd, then it will always be the case that one of these components has less than $\frac{1}{2}|\mathcal{V}(G)|$ vertices, allowing the use of Theorem 13 to reconstruct $\phi(G, x)$ from $\mathscr{P}(G)$. The details are in the proof of the following corollary, which was proved in [10] using a different approach.

Corollary 3 ([10]). Let $G$ be a disconnected graph on $n$ vertices. If not all components of $G$ has exactly $\frac{n}{2}$ vertices, then $\phi(G, x)$ is reconstructible from $\mathscr{P}(G)$. In particular, if $G$ has more than two components or has an odd number of vertices, then $\phi(G, x)$ is reconstructible from $\mathscr{P}(G)$.

Proof. Let $v$ be a vertex of a component $K$ of $G$ having less than $\frac{n}{2}$ vertices. Then $G-v$ and $G$ have more than $\frac{n}{2}$ common eigenvalues. By Theorem 13, this fact is inferable from $\mathscr{P}(G)$, which leads to the reconstruction of $\phi(G, x)$ from $\mathscr{P}(G)$.

Clearly if a disconnected graph $G$ has more than two components or has an odd number of vertices, then $G$ must have such a component $K$.

Note that if $G$ is known to be disconnected, then its characteristic polynomial can be reconstructed from $\mathscr{P}(G)$ [32]. However, we need to stress here that the result of Corollary 3 is true irrespective of whether $G$ is known to be disconnected. Only disconnected graphs having two components, each having $\frac{n}{2}$ vertices, are left out by Corollary 3 .

The results of this paper allow us to include more disconnected graphs than those described in Corollary 3, however. This corollary states that only disconnected graphs having two components with an equal number of vertices may possibly not have their characteristic polynomials reconstructed from their polynomial deck. Let $G$ be one such disconnected graph on $n=2 k$ vertices, whose two components $G_{1}$ and $G_{2}$ have $k$ vertices each. Clearly, every vertex deleted subgraph of $G$ will have at least $k$ eigenvalues in common with those of $G$. Thus, our main result, Theorem 13, will only be inapplicable to $G$ if it so happens that every vertex deleted subgraph of $G$ has exactly $k$ eigenvalues in common with those of $G$.

However, by Theorem 5 , if either of the components of $G$ has an eigenvector associated with one of its eigenvalues $\lambda$ with a zero entry in its $v$ th position, then this eigenvalue will also be present in $G-v$, and hence $G$ will have more than half of its eigenvalues shared by those of $G-v$. This will allow the reconstruction of $\phi(G, x)$ from $\mathscr{P}(G)$ by applying Theorem 13.

Consequently, among all disconnected graphs, only disconnected graphs having two components $G_{1}$ and $G_{2}$ of equal order, where both $G_{1}$ and $G_{2}$ have no zeros in their eigenvectors, may possibly be counterexamples to the polynomial reconstruction conjecture. Such graph components with no zeros in their eigenvectors are called omnicontrollable in [17].

We thus conclude our paper with the following remarkable result that strengthens Corollary 3.

Theorem 14. Let $G$ be a disconnected graph with polynomial deck $\mathscr{P}(G)$. Then $\phi(G, x)$ is reconstructible from $\mathscr{P}(G)$, except possibly if $G$ has two components of equal order, both of which are omnicontrollable graphs.

Funding: This research received no external funding.

Acknowledgments: The author would like to thank the anonymous referees for their useful comments on an earlier version of this paper, which resulted in a greatly improved version.

Conflicts of Interest: The author declares no conflict of interest.

\section{References}

1. Cvetković, D. On the reconstruction of the characteristic polynomial of a graph. Discret. Math. 2000, 212, 45-52. [CrossRef]

2. Farrugia, A.; Gauci, J.B.; Sciriha, I. Non-Singular Graphs With a Singular Deck. Discret. Appl. Math. 2015, 202, 50-57. [CrossRef]

3. Schwenk, A.J. Spectral reconstruction problems. Ann. N. Y. Acad. Sci. 1979, 328, 183-189. [CrossRef] 
4. Simić, S.K. A note on reconstructing the characteristic polynomial of a graph. In Fourth Czechoslovakian Symposium on Combinatorics, Graphs and Complexity; Nešetřil, J., Fiedler, M., Eds.; Combinatorics, Graphs and Complexity; Elsevier Science Publishers B.V.: Amsterdam, The Netherlands, 1992; pp. 315-320.

5. Simić, S.K.; Stanić, Z. On the polynomial reconstruction of graphs whose vertex-deleted subgraphs have spectra bounded from below by -2. Linear Algebra Its Appl. 2008, 428, 1865-1873. [CrossRef]

6. Cvetković, D.; Lepović, M. Seeking counterexamples to the reconstruction conjecture for the characteristic polynomial of graphs and a positive result. Bull. Acad. Serbe Sci. Arts Cl. Sci. Math. Nat. Sci. Math. 1998, 116, 91-100.

7. Simić, S.K.; Stanić, Z. The polynomial reconstruction of unicyclic graphs is unique. Linear Multilinear Algebr. 2007, 55, 35-43. [CrossRef]

8. Cvetković, D.; Gutman, I. The reconstruction problem for characteristic polynomials of graphs. Univ. Beograd. Publ. Elektrotehn. Fak. Ser. Mat. Fiz. 1975, 498-541, 45-48.

9. Sciriha, I. Polynomial reconstruction and terminal vertices. Linear Algebra Its Appl. 2002, 356, 145-156. [CrossRef]

10. Sciriha, I.; Formosa, J. On polynomial reconstruction of disconnected graphs. Util. Math. 2003, 64, 33-44.

11. Cardoso, D.M.; Sciriha, I.; Zerafa, C. Main eigenvalues and ( $\kappa, \tau)$-regular sets. Linear Algebra Its Appl. 2010, 432, 2399-2408. [CrossRef]

12. Cvetković, D.; Rowlinson, P.; Stanić, Z.; Yoon M.G. Controllable Graphs. Bull. Cl. Des Sci. Math. Nat. 2011, 143, 81-88.

13. Farrugia, A. On Strongly Asymmetric and Controllable Primitive Graphs. Discret. Appl. Math. 2016, 211, 58-67. [CrossRef]

14. Powers, D.L.; Sulaiman, M.M. The Walk Partition and Colorations of a Graph. Linear Algebra Its Appl. 1982, 48, 145-159. [CrossRef]

15. Farrugia, A. On Pseudo Walk Matrices. Discret. Math. Lett. 2019, 1, 8-15.

16. Farrugia, A. The rank of Pseudo walk matrices: Controllable and recalcitrant pairs. Open J. Discret. Appl. Math. 2020, 3, 41-52. [CrossRef]

17. Farrugia, A.; Sciriha, I. Controllability of undirected graphs. Linear Algebra Its Appl. 2014, 454, 138-157. [CrossRef]

18. Godsil, C. Controllable Subsets in Graphs. Ann. Comb. 2012, 16, 733-744. [CrossRef]

19. Rowlinson, P. The Main Eigenvalues of a Graph: A survey. Appl. Anal. Discret. Math. 2007, 1, 445-471. [CrossRef]

20. Horn, R.A.; Johnson, C.R. Matrix Analysis, 2nd ed.; Cambridge University Press: Cambridge, UK, 2013.

21. Rahmani, A.; Meng, J.; Mesbahi, M.; Egerstedt, M. Controllability of multi-agent systems from a graph-theoretic perspective. SIAM J. Control Optim. 2009, 48, 162-186. [CrossRef]

22. Fowler, P.W.; Pickup, B.T.; Todorova, T.Z.; De Los Reyes, R.; Sciriha, I. Omni-conducting Fullerenes. Chem. Phys. Lett. 2013, 568-569, 33-35. [CrossRef]

23. Sciriha, I.; Farrugia, A. From Nut Graphs to Molecular Structure and Conductivity; Mathematical Chemistry Monographs; University of Kragujevac: Kragujevac, Serbia, 2021.

24. Debono, M. Threshold Graphs as Models of Real-World Networks. Master's Thesis, University of Malta, Msida, Malta, 2012.

25. Marcus, M.; Minc, H. A Survey of Matrix Theory and Matrix Inequalities; CBMS Regional Conference Series in Mathematics; Allyn and Bacon, Inc.: Boston, MA, USA, 1964; Volume 115.

26. Sciriha, I. Graphs with a common eigenvalue deck. Linear Algebra Appl. 2009, 430, 78-85. [CrossRef]

27. Parter, S. On the eigenvalues and eigenvectors of a class of matrices. J. Soc. Indust. Appl. Math. 1960, 8, 376-388. [CrossRef]

28. Clarke, F.H. A graph polynomial and its application. Discret. Math. 1972, 3, 305-315. [CrossRef]

29. Cvetković, D.; Rowlinson, P.; Simić, S.K. An Introduction to the Theory of Graph Spectra; Cambridge University Press: Cambridge, UK, 2009.

30. Aigner, M. A Course in Enumeration; Springer: Berlin, Germany; New York, NY, USA, 2007.

31. Farrugia, A. On the Spectra and Walks of Molecular and Controllable Graphs. Ph.D. Thesis, University of Malta, Msida, Malta, 2016.

32. Sciriha, I. Polynomial reconstruction: Old and new techniques. Rend. Del Semin. Mat. Messina 2002, 8, $163-179$. 